\title{
Effect of External Charges on the Quantum Superconductor-Insulator Transition in a Josephson-Junction Array.
}

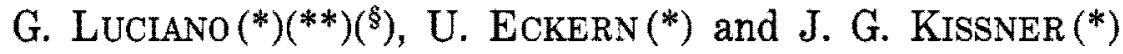 \\ (*) Institut für Physik, Universität Augsburg - D-86135 Augsburg, Germany \\ (**) Dipartimento di Scienze Fisiche, Universita' di Napoli and INFM \\ Mostra d'Oltremare Pad. 19, I-80125 Napoli, Italy
}

PACS. 74.50 $+r$ - Proximity effects, weak links, tunneling phenomena, and Josephson effects. PACS. 74.60Ge - Flux pinning, flux creep, and flux-line lattice dynamics.

PACS. 74.76-w - Superconducting films.

\begin{abstract}
We study the influence of a uniform background of external charges on the dynamics of a vortex moving in a quantum (zero temperature) Josephson-junction array, in the limit in which the capacitance between islands, $C_{1}$, is much larger than the ground capacitance. For large Josephson coupling, $E_{\mathrm{J}} \gg E_{\mathrm{C}}=e^{2} / 2 C_{1}$, we confirm the continuum limit result for the vortex mass, both in the absence and in the presence of the external charges. Upon decreasing $E_{\mathrm{J}} / E_{\mathrm{C}}$, the vortex mass vanishes at a critical value, which can be taken as an indicator of the superconductor-insulator phase transition. The phase diagram, as a function of the external charge, is determined on the basis of a small $\alpha\left(\alpha^{2}=E_{\mathrm{C}} / 8 E_{\mathrm{J}}\right)$ expansion. We find, in particular, that external charges significantly stabilise the superconducting phase.
\end{abstract}

High-quality arrays of Josephson junctions of very small capacitances offer a unique opportunity to test models of quantum statistical mechanies. It has been found [1-3] that such an array can undergo a superconductor-insulator transition at low temperature, when the Coulomb charging energy $E_{\mathrm{C}}$ becomes comparable with the Josephson coupling energy $E_{\mathrm{J}}$ $\left(\alpha=\sqrt{E_{\mathrm{C}} / 8 E_{\mathrm{J}}}-1\right)$. The transition has been studied numerically and analytically in [4], using a description in terms of charges and vortices. It can be analysed from the superconducting side, in particular, by studying the dynamical properties of a single vortex added to the array: a mass can be attributed to the vortex [5], which vanishes at the transition [4]. In addition, external charges added to the islands by a gate voltage induce frustration, thereby stabilising the superconducting phase. This effect was studied in detail for a short-ranged Coulomb interaction $[6,7]$.

In this article, we study the zero-temperature phase diagram and the quantum vortex dynamics in the presence of a uniform static background of offset charges $q$, in the limit of a long-ranged Coulomb interaction (which is relevant for some of the experimentally

(\$) E-mail: Gaetano.Luciano@physik.uni-augsburg.de. 
fabricated arrays). Our method preserves two essential features of the model: the discreteness of charge transfer (by Cooper pairs) between islands, and the invariance of the system, when all external charges are increased by the same multiple of $2 e$, leading to the expected periodicity of all physical quantities.

We consider a square array of Josephson junctions (JJA) which is described by the Hamiltonian

$$
\mathscr{K}=\frac{1}{2}(2 e)^{2} \sum_{i j}\left(n_{i}-q_{i}\right) \mathscr{G}_{i j}^{-1}\left(n_{j}-q_{j}\right)-E_{J} \sum_{(i, j)} \cos \left(\varphi_{i}-\varphi_{j}\right) .
$$

The index $i=\left(i_{x}, i_{y}\right)$ labels the islands, which are characterised by the phases of the superconducting order parameter, $\left\{\varphi_{i}\right\}$, and their conjugate variables, which are the charges, $\left\{2 e n_{i}\right\}:\left[n_{k}, \phi_{l}\right]=-i \delta_{k l}$. We also introduced static external charges, $\left\{2 e q_{i}\right\}$, on each island. $G_{i j}{ }^{-1}$ is the inverse of the capacitance matrix; we take only the ground capacitance, $C_{0}$, and the capacitance between neighbouring islands, $C_{1}\left(E_{\mathrm{C}} \equiv e^{2} / 2 C_{1}\right)$, into account, and assume below that $C_{1} \gg C_{0}$.

In the superconducting phase the partition function can be expressed as a collection of vortex-antivortex pairs of vorticity $v_{i \tau}$ (the vorticity at each space-time point can take the values 0 or \pm 1$)$ [2]. A cumulant expansion yields the partition function in the following form [4]:

$$
\mathcal{Z}=\mathscr{Z}_{n} \sum_{\left\{v_{j \tau}\right\}} \exp \left[-\widetilde{S}_{0}-\widetilde{S}_{1}\right]
$$

with

$$
\widetilde{S}_{0}=\frac{\pi E_{\mathrm{J}}}{\omega_{\mathrm{p}}} \sum_{i j, \tau} v_{i \tau} G_{i j} v_{j \tau},
$$

and

$$
\widetilde{S}_{1}=\frac{1}{2} \sum_{i j, \tau} \sum_{k l, \tau^{\prime}} \mathcal{Q}_{i j, \tau \tau^{\prime}} \Theta_{i k} \Theta_{j l} \dot{v}_{k \tau} \dot{v}_{l \tau^{\prime}}-i \sum_{i, \tau}\left\langle n_{i \tau}\right\rangle \Theta_{i j} \dot{v}_{j \tau}
$$

where $\omega_{\mathrm{p}}=\sqrt{8 E_{\mathrm{C}} E_{\mathrm{J}}}$ is the Josephson-plasma frequency (its inverse is chosen to define the time spacing, $\varepsilon=\omega_{\mathrm{p}}^{-1} ; \hbar=1$ ), and $G_{i j}=-\ln \left|\boldsymbol{r}_{i}-\boldsymbol{r}_{j}\right|$ is the logarithmic vortex-vortex interaction, with $\boldsymbol{r}_{i}=a\left(i_{x}, i_{y}\right)$. Here, and in the following, a dot means a discrete, dimensionless time derivative, and the lattice constant, $a$, is set equal to one. Furthermore, we defined the phase configuration around a vortex, $\Theta_{i j}=\operatorname{arctg}\left(\left(y_{i}-y_{j}\right) /\left(x_{i}-x_{j}\right)\right)$, and the connected charge-charge correlation function, $\mathcal{Q}_{i j, \tau \tau^{\prime}}=\left\langle\left(n_{i \tau}-q_{i}\right)\left(n_{j \tau^{\prime}}-q_{j}\right)\right\rangle_{\mathrm{c}}$. Averages $\langle\cdot\rangle$ are performed with respect to the charge action

$$
S_{n}=\frac{1}{2} \sum_{i j, \tau \tau^{\prime}}\left(n_{i \tau}-q_{i}\right) \mathscr{M}_{i j, \tau \tau^{\prime}}\left(n_{j \tau^{\prime}}-q_{j}\right),
$$

$\mathscr{Z}_{n}=\operatorname{Tr}\left[\exp \left[-S_{n}\right]\right]$, and the matrix $\mathscr{M}$ is defined by $[4]$

$$
\mathscr{M}_{i j, \tau \tau^{\prime}}=\frac{4 E_{\mathrm{C}}}{\pi \omega_{\mathrm{p}}} V_{i j} \delta_{\tau, \tau^{\prime}}+\frac{\omega_{\mathrm{p}} G_{i j}}{2 \pi E_{\mathrm{J}}}\left(2 \delta_{\tau, \tau^{\prime}}-\delta_{\tau, \tau^{\prime}+\varepsilon}-\delta_{\tau, \tau^{\prime}-\varepsilon}\right),
$$

where $V_{i j}=2 \pi C_{1} \mathscr{G}_{i j}^{-1}$; the range of this quantity is $\lambda=\sqrt{C_{1} / C_{0}}$, provided $\lambda>1$. For illustration, we give the Fourier transform of $\mathscr{M},(i, \tau) \rightarrow(\boldsymbol{k}, \omega)$, in the long-wavelength low- 
frequency limit

$$
\mathscr{M}_{k \omega}=\frac{1}{E_{\mathrm{J}}}\left(\frac{\omega^{2} / \omega_{\mathrm{p}}^{2}}{k^{2}}+\frac{1}{\lambda^{-2}+k^{2}}\right)
$$

Adding a single vortex with trajectory $\boldsymbol{r}(\tau)$ and configuration $v_{i \tau}=\delta\left(\boldsymbol{r}_{i}-\boldsymbol{r}(\tau)\right)$ to the array, one derives an effective vortex action, which allows to identify the vortex mass tensor as follows:

$$
M_{\alpha \beta}\left[\boldsymbol{r}(\tau)-\boldsymbol{r}\left(\tau^{\prime}\right), \tau-\tau^{\prime}\right]=\sum_{i j} \nabla_{\alpha} \Theta\left(\boldsymbol{r}(\tau)-\boldsymbol{r}_{i}\right) \mathcal{E}_{i j, \tau \tau^{\prime}} \nabla_{\beta} \Theta\left(\boldsymbol{r}_{j}-\boldsymbol{r}\left(\tau^{\prime}\right)\right)
$$

Clearly, the charge-charge correlation function determines various aspects of the vortex dynamics [8,4]; for example, considering a constant and small vortex velocity, the vortex mass is

$$
M_{\mathrm{v}}=\omega_{\mathrm{p}}^{-1} \sum_{\tau} M_{x \propto}(0, \tau)=\frac{1}{2} \int \frac{\mathrm{d}^{2} k}{k^{2}} \mathcal{L}(\boldsymbol{k}, \omega=0) .
$$

In order to evaluate $\mathcal{Q}_{i j, \tau}$, and $\left\langle n_{i \tau}\right\rangle$ explicitly, we perform a Poisson resummation. The averages are expressed in terms of discrete auxiliary fields, $\phi_{i \tau}$, that run over all integers, $\phi_{i \tau}=0, \pm 1, \pm 2, \ldots$. The resulting expressions for $\left\langle n_{i \tau}\right\rangle$ and $\mathcal{Z}_{i j, \tau}$ are the following:

$$
\left\langle n_{i \tau}\right\rangle=q_{i}+2 \pi i \sum_{j \tau^{\prime}} \mathscr{M}_{i j, \tau \tau^{\prime}}^{-1}\left\langle\phi_{j \tau^{\prime}}\right\rangle
$$

and

$$
\mathcal{Q}_{i j, \tau \tau^{\prime}}=\mathscr{M}_{i j, \tau \tau^{\prime}}^{-1}-(2 \pi)^{2} \sum_{k l, \tau_{1} \tau_{2}} \mathscr{M}_{i k, \tau_{1}}^{-1}\left\langle\phi_{k \tau_{1}} \phi_{l \tau_{2}}\right\rangle_{\mathrm{c}} \mathscr{M}_{l, \tau_{2} \tau^{*}}^{-1}
$$

where the new average $\langle\cdot\rangle$ is performed with the action

$$
S_{\phi}=2 \pi^{2} \sum_{i, \tau \tau^{\prime}} \phi_{i \tau} \mathscr{M}_{i j, \tau \tau^{\prime}}^{-1} \phi_{j \tau^{\prime}}-2 \pi i \sum_{i \tau} q_{i} \phi_{i \tau} .
$$

Clearly, $\left\langle n_{i \tau}\right\rangle=q$ when the external charge is homogeneous (which we consider from now on). The advantage of introducing the $\phi$-fields is that, in the limit $\lambda \gg 1$, they interact through the short-ranged kernel

$$
\mathscr{M}_{i j, \tau^{\prime}}^{-1}=\frac{1}{16 \alpha}\left(-\nabla^{2}\right) \exp \left[-\omega_{\mathrm{p}}\left|\tau-\tau^{\prime}\right|\right]
$$

where $\nabla^{2}$ denotes the Laplacian on the lattice. Since $\mathscr{M}_{k, w=0}^{-1}=k^{2} / 8 E_{\mathrm{C}}$, it is easily seen that the first term to the right of eq. (11) gives the classical contribution [5] for the vortex mass, $M_{\mathrm{ES}}=\pi^{2} / 4 E_{\mathrm{C}}$. Furthermore, it is apparent that the correlation function $\mathscr{R}=\left\langle\phi_{i \tau} \phi_{j \tau^{\prime}}\right\rangle_{\mathrm{c}}$ is exponentially small for $\alpha \rightarrow 0$, confirming the classical result for small $\alpha$, while for $\alpha \rightarrow \infty$, i.e. in the insulating regime, the $\phi$-fields can be treated as continuous variables: thus $\mathscr{K}=$ $=\mathscr{M} / 4 \pi^{2}$ in this limit, and $\mathcal{Q} \equiv 0$. In between, at a critical value $\alpha_{\mathrm{c}}$, the model is expected to have a phase transition of the roughening type (this is clear when we replace the exponential time dependence in eq. (13) by a $\delta$-function $[9,10])$, such that for small $k, \mathscr{K}_{k, \omega=0} \sim k^{-2}$ for $\alpha>\alpha_{\mathrm{c}}$, while $\mathscr{R}_{k, \omega=0}$ approaches a constant for $\alpha<\alpha_{\mathrm{c}}$, which we denote by $\xi^{2} \cdot 2 \alpha / \pi^{2} \omega_{\mathrm{p}}$. The dimensionless correlation length, $\xi$, is expected to diverge at the transition; it also characterises the stiffening of the the spin-wave spectrum [4], $\omega_{\mathrm{p}}^{2} \rightarrow \omega_{\mathrm{p}}^{2}\left(1+\xi^{2} k^{2}\right)$, and, assuming that the charge-charge correlation function is of the form given by the SCHA 
approximation, it determines the vortex mass according to [4]

$$
M_{\mathrm{v}} / M_{\mathrm{ES}}=\left(4 \pi \xi^{2}\right)^{-1} \ln \left(1+4 \pi \xi^{2}\right) .
$$

Thus, $M_{\mathrm{v}} \rightarrow M_{\mathrm{ES}}$ for $\xi \rightarrow 0$, and $M_{\mathrm{v}} \rightarrow 0$ for $\xi \rightarrow \infty$.

To be precise, we define the connected correlation function, $\mathscr{R}_{m n, v}=\left\langle\phi_{i \tau} \phi_{j \tau}\right\rangle-$ $-\left\langle\phi_{i \tau}\right\rangle\left\langle\phi_{j \tau^{\prime}}\right\rangle$, where $j=i+m \hat{x}+n \hat{y}, \tau^{\prime}=\tau+\nu \varepsilon(m, n, v$ are integers; clearly, this quantity is independent of $(i, \tau))$. For finite but small $\alpha, \mathscr{R}_{m n, \nu}$ is computed by a small $\alpha$ expansion, in the same spirit as the low-temperature expansion [11]. Then, only a few sites are "excited», that is we can compute the average by including only those configurations that have a small number of excitations. For example, $\mathscr{K}_{00,0}$ is given by the following expression $\left(s=\exp \left[-\pi^{2} / 4 \alpha\right]\right)$ :

$$
\begin{aligned}
& \mathscr{R}_{00,0}=2 \cos (2 \pi q) s^{2}+8 \cos (4 \pi q) s^{3}+[-8-12 \cos (4 \pi q)+ \\
& \quad+36 \cos (6 \pi q)+8 \cos (8 \pi q)] s^{4}+O\left(s^{5}\right)+2 s^{4} \sum_{v \neq 0} T_{v}^{+}+O\left(s^{4(1-1 / 4 e)}\right),
\end{aligned}
$$

where $T_{v}^{+}=\left(s^{4 / \exp [|v| 1}-1\right) \cos (4 \pi q)+\left(s^{-4 / \exp [|v|]}-1\right)$, and $e=2.71 \ldots$ The terms with integer exponents of $s$ are related to the excitations in the space directions, whereas those with non-integer exponents originate from excitations in the time direction. The latter do not play a significant role in the calculation of the correlation length, and we neglect them in the following. Furthermore, $\mathscr{K}_{m n, v}$ is strongly peaked at $m=n=v=0$, and falls off rapidly by increasing the space or time distance, at least for not too large an external charge, i.e. we may restrict ourselves to a few terms $\left(\mathscr{K}_{00,0}, \mathscr{K}_{10,0}, \mathscr{K}_{11,0}, \mathscr{K}_{20,0}\right)$ when calculating the correlation length, $\xi^{2}=\left(\pi^{2} / 2 a\right) \sum_{m n, v} \mathscr{R}_{m n, v}$. Explicitly, we obtain

$\xi^{2}=\frac{\pi^{2}}{2 \alpha}\left\{2 \cos (2 \pi q) s^{2}+16 \cos (4 \pi q) s^{3}+\right.$

$$
\left.+[-20 \cos (4 \pi q)+108 \cos (6 \pi q)+32 \cos (8 \pi q)] s^{4}+\ldots\right\} .
$$

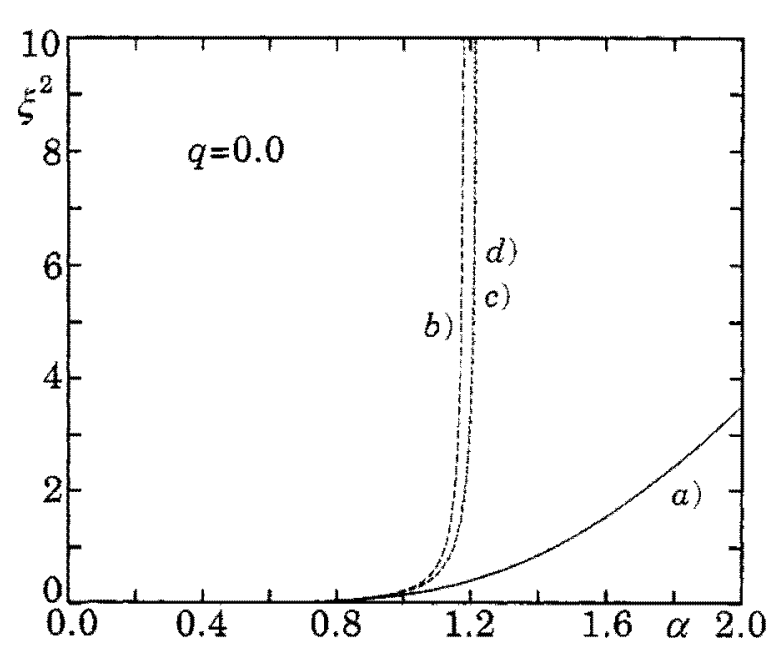

Fig. 1.

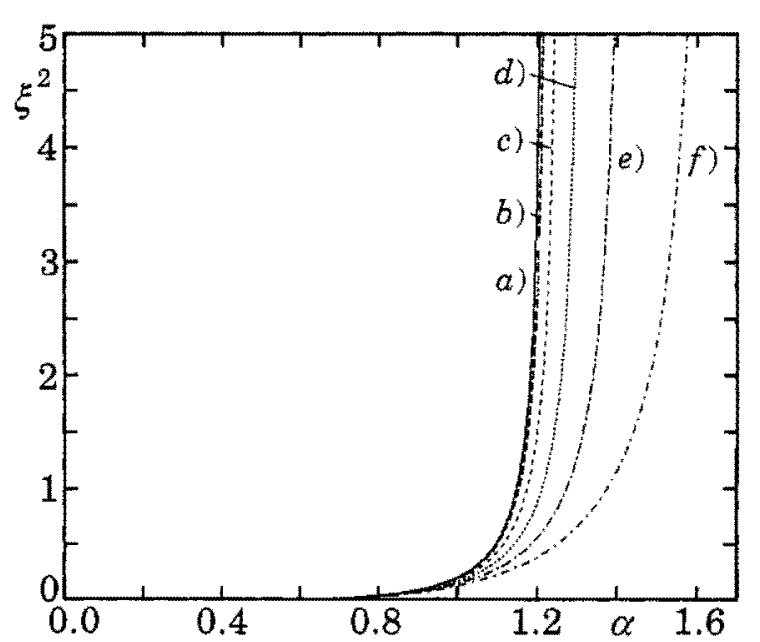

Fig. 2.

Fig. 1. - Squared correlation length $v s . \alpha$ in the absence of external charges, based on eq. (16) (curve a)) and for the three Padé approximations of this expression, $P_{1}^{0}(s), P_{2}^{0}(s)$, and $P_{1}^{1}(s)$ (curves $\left.b\right)-d$ ), respectively).

Fig. 2. - Squared correlation length $v s . \alpha$, on the basis of $P_{1}^{1}(s)$, for different values of the external charge. From a) to $f$ ), $q$ increases from 0.00 to 0.05 in steps of 0.01 . 


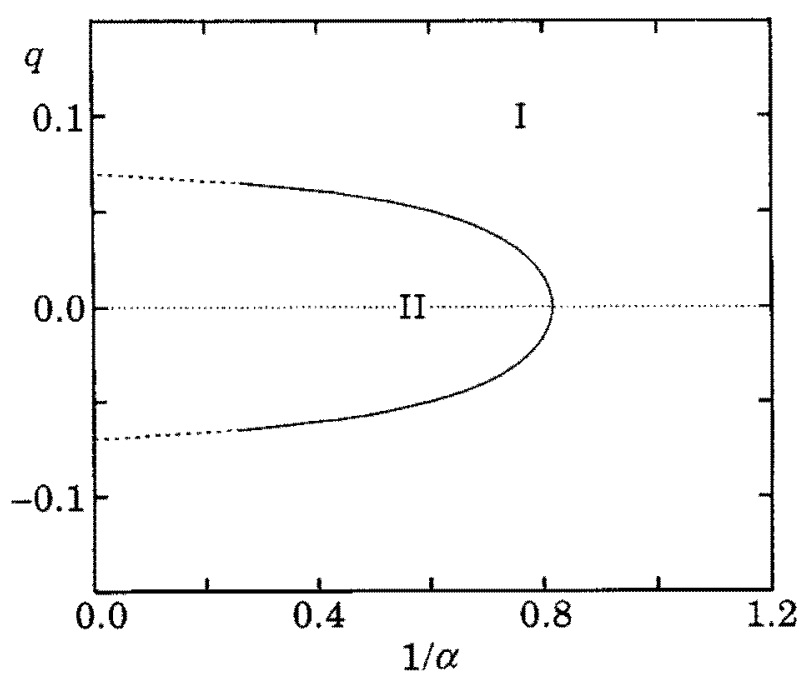

Fig. 3.

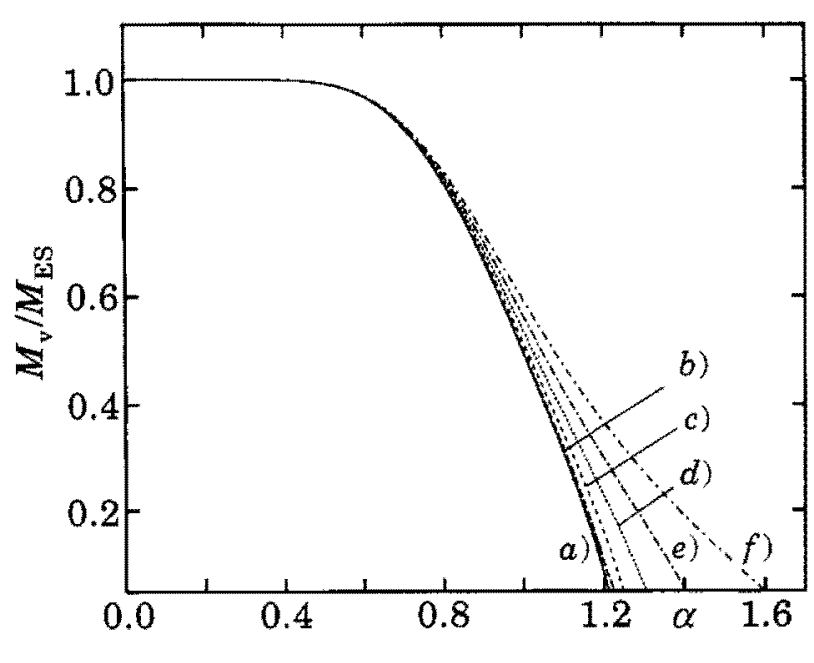

Fig. 4.

Fig. 3. - Phase diagram as a function of $1 / \alpha$ and $q$. I: superconducting phase, II: insulating phase. The range of $\alpha$-values where our approach is less reliable is shown as a dashed line.

Fig. 4. - Vortex mass (normalised to $M_{\mathrm{ES}}$ ) vs. $\alpha$, as calculated from eq. (14), for the same $q$-values as in fig. 2.

This result is of limited usefulness (see fig. 1, curve a)); however, a Padé approximation immediately leads to the desired divergence at a critical value of $\alpha$ and, fortunately, the behaviour is qualitatively similar for the different Padé approximations, $P_{1}^{0}(s), P_{2}^{0}(s)$, and $P_{1}^{1}(s)$ (fig. 1). In the following, we work with $P_{1}^{1}(s)$. The critical value is close to 1.2 for $q=0$, and moves quickly to higher $\alpha$ with increasing $q$ (fig. 2). From these results, it is straightforward to determine the phase diagram and the vortex mass. The results are shown in fig. 3 and 4 , respectively.

In summary, we studied the zero-temperature phase diagram and the quantum dynamics of a vortex in a Josephson-junction array with a uniform static background of offset charges $q$, in the limit of a long-ranged Coulomb interaction, by an analytical method similar to a lowtemperature expansion. We have confirmed that the vortex mass tends to $M_{\mathrm{ES}}$, independent of $q$, in the limit of a dominant Josephson coupling energy $(\alpha \rightarrow 0)$, unlike other approaches which predict an unphysical, lower value in the classical limit [4]. Furthermore, the phase boundary for $q=0$ is found to be at $\alpha_{c} \simeq 1.2$, unlike earlier studies which gave $\approx 1.05$ (SCHA approximation), $\approx 0.79$ (duality arguments), $\simeq 0.46$ (Monte Carlo results) [4], and $\simeq 0.5$ (variational approach [3]). In view of the differences in the methods which were used, we are at present not able to resolve these discrepancies (though it must be noted that the last values are closest to the one found experimentally; see, e.g.,[12] for a-theoretical and experimental-review). For a finite offset charge, we find the disordered (insulating) region to be strongly suppressed, in particular more strongly than for a short-ranged Coulomb interaction. However, some indications of this tendency are already apparent in studies on small systems [4]. From the form of the phase diagram, see fig. 3, we speculate that the transition disappears when the magnitude of the offset charge is strong enough.

The question of the effect of random offset charges, presumably most important for experiments on Josephson-junction arrays, remains an open problem.

$$
* * *
$$

We thank A. Tagliacozzo, R. Fazio and A. van OtTERLo for valuable discussions. Part of this work was performed at the Institute for Scientific Interchange, Torino, whose hospitality is gratefully acknowledged. 


\section{REFERENCES}

[1] VAN der ZaNT H. S. J., Fritschy F. C. and MooIJ J. E., Europhys. Lett, 19 (1992) 541.

[2] FAZIo R. and SchöN G., Phys. Rev. B, 43 (1991) 5307.

[3] Kissner J. G. and Eckern U., Z. Phys. B, 91 (1993) 155.

[4] Fazio R., van Otterlo A. and SchöN G., Europhys. Lett., 25 (1994) 453; van OtTerlo A., Ph. D. thesis, University of Karlsruhe, May 1994 (unpublished); VAN OTTERLO A., FAzIO R. and SchöN G., Physica B, 203 (1994) 504.

[5] Eckern U. and Schmid A., Phys. Rev. B, 10 (1989) 6641.

[6] Roddick E. and Stroud D. H., Phys. Rev. B, 48 (1993) 16600.

[7] van Otterlo A., Wagenblast K.-H., Fazio R. and Schön G., Phys. Rev. B, 48 (1993) 3316; Bruder C., Fazio R., Kampf A. P., van Otterlo A. and Schön G., Phys. Scr., T 42 (1992) 159; VAn Otterlo A. and Wagenblast K.-H., Phys. Rev. Lett., 72 (1994) 3598.

[8] ECKern U. and Sonin E. B., Phys. Rev. B, 47 (1993) 505.

[9] Chui S. T. and Weeks J. D., Phys. Rev. B, 14 (1976) 4978.

[10] VAN BeIJeren H. and Nolden I, in Structure and Dynamics of Surfaces II, edited by W. SChommers and P. von Blanckenhagen (Springer, Heidelberg) 1987, p. 259; B. Nienhuis, in: Phase Transitions and Critical Phenomena, edited by C. Domb and J. L. LeBowitz, Vol. 11 (Academic, London) 1987, p. 1.

[11] DomB C., in Phase Transitions and Critical Phenomena, edited by C. DoMB and M. S. GREEN, Vol. 3 (Academic, London) 1974, p. 225.

[12] Fazio R., van Otterlo A., Schön G., van der Zant H. S. J. and Moois J. E., Helv. Phys. Acta, 65 (1992) 228. 